\title{
Reseña del libro: Actitudes hacia las matemáticas en la resolución de problemas y su relación con la investigación propia
}

Sabino Ariel Olivar Molina ${ }^{1}$

\section{Información de artículo:}

Recibido: 10/10/2019

Aprobado: $30 / 10 / 2019$

\section{Palabras claves:}

Actitudes hacia las matemáticas; resolución de problemas; Creencias en la resolución de problemas.

\section{Keywords:}

Attitudes towards Mathematics; Problem resolution;

Problem solving beliefs.

\section{Resumen}

El libro sobre actitudes hacia las matemáticas en la resolución de problemas y su relación con la investigación propia trata de dinamizar los conceptos de emociones, creencias y actitudes, así como su relación con el aprendizaje de las matemáticas en la resolución de problemas. Además, se presentan experiencias de investigación: (1) identificar las actitudes hacia las matemáticas y su relación con las variables género y etnia; y (2) creencias en la resolución de problemas matemáticos. El libro finaliza un referente teórico sobre metodologías de resolución de problemas para los procesos de investigación en educación matemática y sus didácticas para su fomento en la formación de profesores de matemática.

\section{Book Review: Attitudes towards Mathematics in problem solving and its relationship with own research}

\section{Abstract}

The book on attitudes towards mathematics in solving problems and their relationship with their own research tries to energize the concepts of emotions, beliefs and attitudes, as well as their relationship with the learning of mathematics in problem solving. In addition, research experiences are presented: (1) identify attitudes towards mathematics and their relationship with the variables gender and ethnicity; and (2) beliefs in solving mathematical problems. The book finalizes a theoretical reference on problem solving methodologies for mathematical education research processes and their didactics for their promotion in the training of mathematics teachers.

\footnotetext{
${ }^{1}$ Máster en Didáctica de las Matemáticas. Profesor de la Universidad de las Regiones Autónomas de la Costas Caribe Nicaragüense. Email: sabino.olivar@uraccan.edu.ni, (1): https://orcid.org/oooo-0003-0505-8757
} 


\section{Introducción}

Actitudes hacia las matemáticas en la resolución de problemas y su relación con la investigación propia, trata de dinamizar los conceptos de emociones, creencias y actitudes, así como su relación con el aprendizaje de las matemáticas en la resolución de problemas.

\section{Reseña del libro}

El libro "Actitudes hacia las matemáticas en la resolución de problemas y su relación con la investigación propia" del año 2019, de la Editorial URACCAN con ISBN: 97899964-23-15-4, publicado en la ciudad de Managua, Nicaragua. El libro esta organizado en cinco capítulos, que expresan lo siguiente:

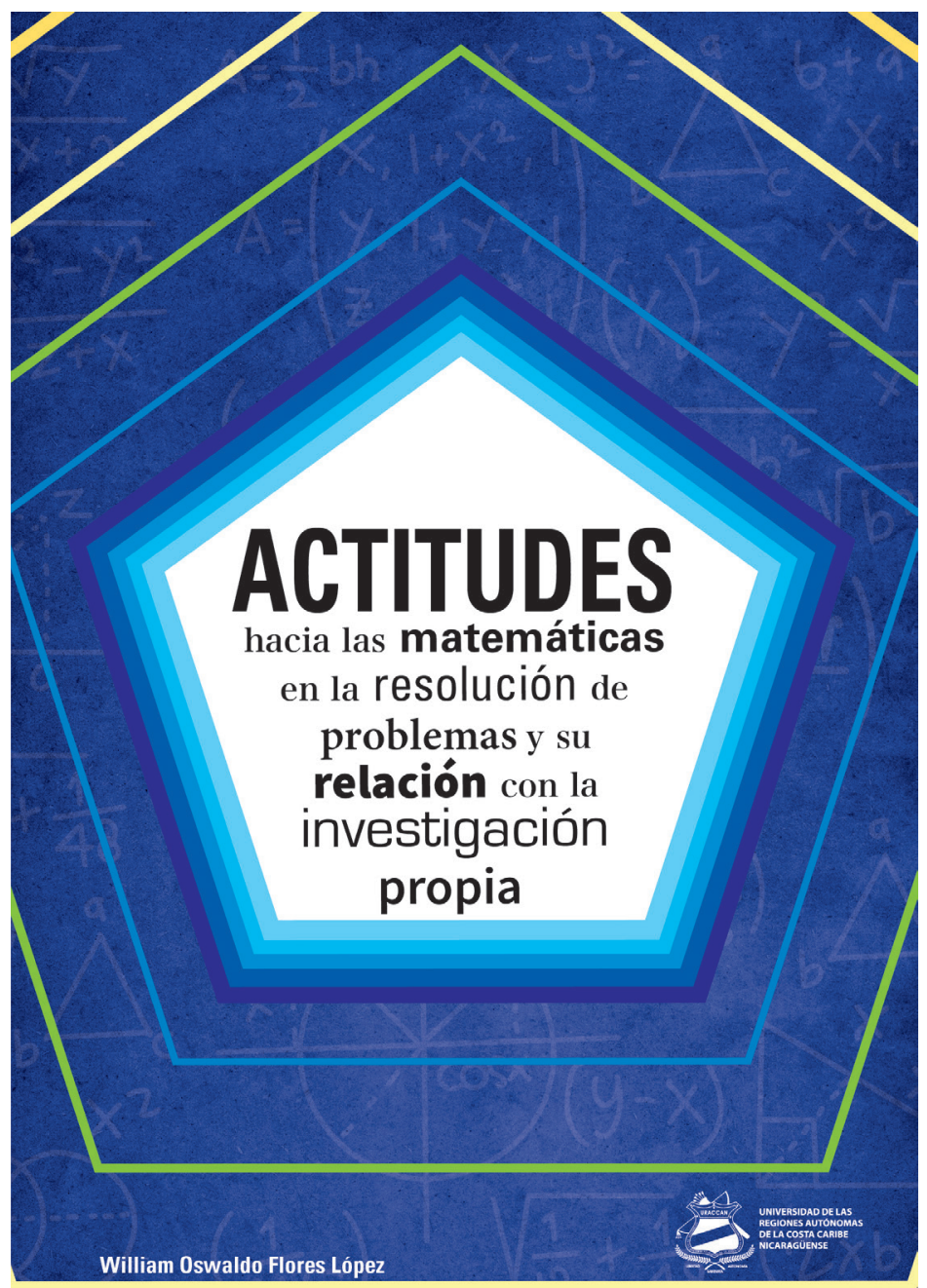


En el primer capítulo "actitudes hacia las matemáticas" ha partido del hecho de que un objetivo en la enseñanza y aprendizaje de las matemáticas es que el estudiantado desarrolle emociones, creencias y actitudes que aumente sus probabilidades de utilizar con éxito en las matemáticas (Flores \& Auzmendi, 2018). Entonces, se abordan aspectos específicos como el dominio afectivo; emociones; creencias; actitudes; factores que influyen en las actitudes hacia las matemáticas; problemas en la enseñanza, aprendizaje y evaluación de las matemáticas; actitud hacia las matemáticas; y actitudes matemáticas.

En el segundo capítulo se hace una aproximación a las tipologías de escalas que miden las actitudes y creencias hacia las matemáticas en la resolución de problema. Además, se desarrolla teóricamente experiencias de construcción y adecuación de instrumentos de medidas como las escalas de Aiken y Dreger (1961), Fennema y Sherman (1976), Gairin (1992), Auzmendi (1992), Quiles (1993), Bazán y Sotero (1998), Cazorla, Silva y Brito (1999), Tapia y Marsh (2004), Muñoz y Mato (2008), Alemany y Lara (2010), Adelson y McCoach (2011), Estrada y Diez-Palomar (2011), González-Pineda et al. (2012) y Flores y Auzmendi (2015). También, se presenta la conceptualización de fiabilidad y validez de las escalas con el objetivo que el lector o lectora tenga herramientas conceptuales y procedimentales para garantizar la calidad de la medida de los instrumentos de investigación.

En el tercer capítulo, se ejemplifica cómo se pueden identificar actitudes hacia las matemáticas en las variables género y etnia. Se trata de una experiencia de investigación que tiene un abordaje desde la propuesta en marcha de investigación acción intercultural con la utilización de métodos cuantitativos y cualitativos. Los resultados sistematizan los significados propios y compartidos sobre actitud, ansiedad, agrado, confianza, motivación y utilidad hacia las matemáticas. Igualmente, se explica la relación de las actitudes hacia las matemáticas con las variables género y etnia en los procesos de resolución de problemas.

En el cuarto capítulo, se trata de analizar las creencias en la resolución de problemas, teniendo de referencia que las creencias se van construyendo y transformando a lo largo de toda la vida, además, que son inherentes en las actitudes. Entonces, se presenta una experiencia de investigación con estudiantes en procesos de formación para maestros de educación primaria, abordada desde la investigación acción intercultural con la utilización de métodos cualitativos como grupo focales y análisis de contenidos; y métodos cuantitativos como el cuestionario de dominio afectivo en la resolución de problemas matemáticos (Caballero-Carrasco \& Guerrero-Barona, 2015). Los resultados muestran conceptos y definiciones propias sobre creencias en la resolución de problemas; enseñanza en la resolución de problemas; aprendizaje en la resolución de problema; y resolución de problemas. Finalmente, se identificaron 


\section{RESEÑA DE LIBRO}

creencias en la resolución de problemas en relación con las variables género, edad, etnia, procedencia y experiencia docente.

La resolución de cualquier problema matemático lleva asociada una situación afectiva para el sujeto implicado, quien pone en juego no solamente prácticas operativas y discursivas para dar una respuesta al problema, sino también moviliza creencias, actitudes, emociones y valores que condicionan, en mayor o menor grado y diferente sentido, la respuesta cognitiva requerida (Godino, 2013, p. 122). Partiendo de este planteamiento, en el quinto capítulo, se presenta las metodologías de resolución de problemas para los procesos de investigación en educación matemática y sus didácticas. Se trata de un referente teórico sobre la investigación propia para la resolución de problemas matemáticos, donde se propone que el dominio afectivo e intercultural es una competencia de hombres y mujeres para controlar y dominar actitudes, creencias y emociones para la toma de decisiones conjuntas en igualdad y equidad de condiciones en la práctica de resolución de problemas matemáticos. Esta metodología de investigación propia para resolución de problemas se puede aplicar en los campos estructurantes: personal-familiar; social-comunitario; profesionalespecializado; científico-tecnológico.

Referencia del libro: Flores, W., O. (2019). Actitudes hacia las matemáticas en la resolución de problemas y su relación con la investigación propia. Managua: Editorial URACCAN. ISBN: 978-99964-23-15-4.

\section{Lista de referencias}

Adelson, J. L. \& McCoach, D. B. (2011). Development and psychometric properties of the math and me survey: Measuring third through sixth graders' attitudes toward mathematics. Measurement and Evaluation in Counseling and Development, 44 (1), 225-247.

Aiken, L. R., \& Dreger, R. M. (1961). The effect of attitude on perfomance in mathematics. Journal of Educational Psychology, 52, 19-24.

Alemany, I., \& Lara, A. I. (2010). Las actitudes hacia las matemáticas en el alumnado de la ESO: un instrumento para su medición. Publicaciones, 40, 49-71.

Auzmendi, E. (1992). Las actitudes hacia las matemáticas-estadística en la enseñanza medias y universitarios. Bilbao: Mensajero. 
Bazán, J., \& Sotero, H. (1998). Una aplicación al estudio de actitudes hacia la matemática en la Unalm. Anales Científicos UNALM, 36, 60-70.

Caballero-Carrasco, A., \& Guerrero-Barona, E. (2015). Un cuestionario sobre dominio afectivo y resolución de problemas de matemáticas. En L. Blanco, J. Cárdenas-Lizarazo \& A. Caballero-Carrasco (Eds.). La resolución de problemas matemáticos en la formación inicial de profesores de primaria (39-57). Cáceres: UNEX.

Cazorla, I. M., Silva, C. B., \& Brito, M. R. (1999). Adaptaçao e validaçao de uma escala de attitudes em relaçao à estatística. Conferencia Internacional Experièncias e Perspec- tivas do Ensino da Estadística (45-58). Florianópolis: Presta.

Estrada, A. y Díez-Palomar, J. (2011). Las actitudes hacia las matemáticas. Análisis descriptivo de un estudio exploratorio centrado en la educación matemática de familiares. Revista de Investigación en Educación Matemática, 9(2), 116-132.

Fennema, E. \& Sherman, J. A. (1976). Mathematics Attitudes Scales: Instruments designed to measure attitudes toward the learning of mathematics by females and males. Journal for Researcher in Mathematics Education, 7(5), 324-326.

Flores, W., O., \& Auzmendi, E. (2015). Análisis de la estructura factorial de una escala de actitud hacia las matemáticas. Aula de Encuentro, 17(1), 45-77.

Flores, W., O., y Auzmendi, E. (2018). Actitudes hacia las matemáticas en la enseñanza universitaria y su relación con las variables género y etnia. Profesorado. Revista de Currículum y Formación de Profesorado, 22(3), 231-251. DOI: https:// doi.org/10.30827/profesorado.v22i3.8000

Gairin, J. (1990). Las actitudes en educación. Un estudio sobre la educación matemática. Barcelona: Boixareau Universitaria.

Godino, J. D. (2013). Indicadores de la idoneidad didáctica de procesos de enseñanza y aprendizaje de las matemáticas. Cuadernos de Investigación y Formación en Educación Matemática, 11, 111-132.

Gónzalez-Pineda, J. A., Fernández-Cueli, M., García, T., Suárez, N., Fernández, E., Tuero- Herrero, E., \& Silva, H. (2012). Diferencias de género en actitudes hacia las mate- máticas en la enseñanza obligatoria. Revista Iberoamericana de Psicología y Salud, 3(1), 55-73.

Muñoz, J., \& Mato, M. (2008). Análisis de las actitudes respecto a las matemáticas en el alumnado de ESO. Revista de Investigación Educativa, 26(1), 209-226. 


\section{RESEÑA DE LIBRO}

Quiles, M. N. (1993). Actitudes matemáticas y rendimiento escolar. Comunicación, Lenguaje y Educación, 18, 115-125.

Tapia, M. \& Marsh, G. E. (2004). An instrument to measure mathematics attitudes. Academic Exchange Quarterly, 8 (2). 